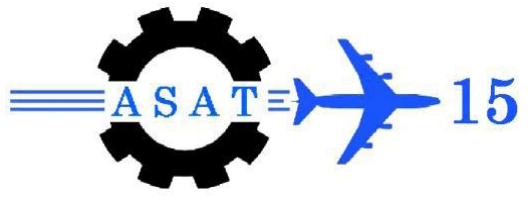

\title{
Experimental Evaluation of the Effect of Accumulator Location on Hydraulic Shock Damping
}

\author{
\{A. M. Gamal, I. Saleh, I. Elsherif*, H. Abdelkader $\}^{\dagger}$
}

\begin{abstract}
One of the challenges in any hydraulic power circuit is the arrangement of the components to utilize significant reliability. This challenge becomes harder with complex circuits or cyclic operation load. The hydraulic circuit of the concrete pump with the s-plane tube is a typical example of a circuit with cyclic operation actuator with high inertia load. This paper investigates experimentally the effect of accumulator location on damping the shock born from cycling operation of an actuator with an inertia load. The investigated circuit mimics the operation of a concrete pump or any system with inertia load. Several locations of accumulator near load and near pump were investigated to find the wave effect on the pump. The results show that an accumulator location in the hydraulic system near the load will minimize the pressure waves that affect pump and cause its quick failure.
\end{abstract}

Keywords: Hydraulic system, accumulator, pressure damping, pressure wave.

\section{Introduction}

Critical operation occurs in resonance situations where excitement by the pump and the natural frequencies of the compressible continuum in the piping system coincide. In many cases, resonance effect can be avoided by proper piping geometry, improving pump performance with respect to the volumetric efficiency or higher fluid friction[1], also can be avoided by changing location of damping element, changing the pre-charged pressure of damper [2], or changing the timing of the system.

In the range of the excitement frequencies of pumps, most of the damper systems are acting as elastic elements (exhibiting spring action) that shift the natural frequency of the hydraulic system away from initially existing resonances.

Pumps deliver a pulsating flow. For safe and reliable operation it is very essential to keep the amplitudes and frequencies of pressure pulsation within close limits. In order to ensure smooth operating conditions and to avoid any secondary vibration effects, the design of damping devices requires taking the whole installation of the system into account[1].

The sudden start/stop of actuators, fast switching valves or reversal motion of high inertia loads may cause a pressure wave that is travel back to the system. This pressure wave can develop peak pressure several times greater than normal working pressure causing objectionable noise or pump failure[2].

ibaelsh@yahoo.com .

$\dagger \quad$ Egyptian Armed Forces, Egypt. 


\section{- The Importance for Designing Safe Piping}

The aims for safe piping design are to avoid:

- Cavitation in the pump or system.

- Super flow at the pump.

- Super load in the pump or system.

- Non admissible pressure pulsations or shacking forces.

Frequencies which may initiate hydraulic or mechanical resonance.[1]

This paper concerned on avoiding the non-admissible pressure pulsations and avoiding the frequencies which may initiate hydraulic or mechanical resonance

\section{- Methods for Pulsation Damping}

Available methods in general are:

a) Reduction of the excitation by the pump and selection of larger pipe size.

b) Avoiding of resonance conditions by adequate piping geometry.

c) Application of damping system with accumulative, dissipative, interference of filtering effects and shifting of the natural frequencies.

Usually, when pulsation analysis has to be performed, there is no chance left for primary measures like a) and b) because pumps and piping systems have already been selected. Accordingly, the only remaining applications are secondary measures like pulsation dampers and / or orifice plates.

Since the best solution for damping pressure pulsation of a low frequency machine like a rock drill machine is a well-supported accumulator installed near the mainline or the T-pipe[3], so a hydraulic membrane accumulator model $\mathrm{GmbH}$ of type OLAER (D-66440 BLIESKASTEL) is used in this paper for this reason.

Accumulators are used for flow enhancement as well as shock absorbing and may act as safety devices to prevent loads from being dropped in case of an engine or pump failure or fluid leak, Also, accumulators improve the reliability and working performance of the oil passage device effectively, so that the oil pump can operate in a stable working state to supply oil to the working oil passage[4].

Accumulators are commonly used in lifts and other equipment, to absorb shocks, which results from a load starting, stopping, or reversal. The energy accumulation capacity of the auxiliary energy-accumulation device changes with the variation of the pressure of hydraulic oil in the device. When the pressure variation increases, the energy accumulation and energy release capability is improved accordingly [5].

Vetter et al.[1], studies the available methods for pulsation damping, The effect of improving the volumetric efficiency on the pressure and frequency damping has been studied, it has been noticed that improving volumetric efficiency for simplex pumps not be an efficient means to reduce pulsations. But the improvement of volumetric efficiency is a simple method to reduce pulsation for multiplex reciprocating pumps. Also, the effect of fluid friction has been studied. It is noticed that increasing fluid friction by viscosity develops remarkable damping effect. As fluid friction is normally not a relevant parameter, nevertheless, piping systems with viscous fluids develop lesser pulsations. 
Mika Ijas [3], Studied the pressure dampers when the damped frequency is low and when they work as a part of a wider system. After theoretical inspection, pressure dampers were simulated and then different dampers were tested with a pure sine wave pressure oscillation and then with the real rock drill. Different sizes of accumulators, a Helmholz resonator, a Tpipe and an inline suppressor were used in tests. He states that the accumulator near the hydraulic main line is not necessarily the best for all applications. The total damping should be better when the natural frequency is the basis of dimensioning. If damping is not desired, it would be useful to try the "short pipe" method.

Kozma[6], focused his work on studying and describing of two known hydraulic impact mechanism one of the impact structures is to be patented. Improvements in the constructive structure and in the operating hydraulic scheme of these impact mechanisms are analyzed. The aim is to simplify the hydraulic scheme and the command and control structure, to minimize the hydraulic parasitic capacities and to attain an adjustable impact frequency. In brief, the goals are to improve the constructive structure of a hydraulic impact device or a hydraulic rotary percussive mechanism controlled by a rotary distributor, hydraulic accumulators, and the operating principle of the mechanism.

He found that it is needed to take into account the effect of the mounting a hydraulic accumulator at the relief of the fluid flow on the discharge line of the impact mechanism to maintain a desired rotational speed and frequency of impacts delivered by the impact or strike piston.

Herein, in this study experimental set up for an electro-hydraulic circuit similar to a part of concrete pump electro-hydraulic circuit has been implemented to study the effect of accumulator location, near load or pump, on the hydraulic system performance under different conditions.

And the effect of changing the pre-charged pressure of accumulator, inserted in the hydraulic circuit, on the pressure pulsation and frequency damping.

Necessary sensors have been added in order to detect the variation of pressure and frequencies of the circuit by sending its signals to a designed Lab View Control and Graphical User Interface program, Also calibrations of measuring devices have been performed.

\section{Experimental Set Up}

In order to specify the attenuation of pressure and frequency pulsation at both sides of fluctuating double acting double-rod hydraulic cylinder and at the nearest point from the pump, an experimental setup depicted in Figure (1) was used. In this setup, a hydraulic bench at which the hydraulic system arrangement is assembled has been used attached with a fixed displacement gear pump with maximum flow rate $(6.05 \mathrm{~L} / \mathrm{min})$ attached to a 40 Liters tank of hydraulic oil type ISO VG 46 of density $(875 \mathrm{~kg} / \mathrm{m} 3)$ and of compressibility $1.4 \times 109 \mathrm{~Pa}$ [7], and operates with an AC electrical motor $(220 \mathrm{~V}, 50 \mathrm{HZ}, 1.5 \mathrm{KW})$.

A relief valve No.(2237 001) manufactured by parker group is used in the hydraulic circuit to limit the maximum operating pressure in the high-pressure lines with operating range (0-55 bar).

The flow pulsation was generated by the switching of a 4/3 electro-hydraulic directional control valve (EHDCV) manufactured by parker group item No.(D1VW20BNJW75) with 
350 bar maximum pressure is used for directing the flow inward and outword a double action double rod hydraulic cylinder item (t2103405) ELWE- Germany manufactured by Bosch group associated with dead loads.

A hydraulic membrane accumulator model $\mathrm{GmbH}$ of type OLAER (D-66440 BLIESKASTEL) with $750 \mathrm{cc}$ capacity has been used to reduce pressure pulsation in the pressure and tank lines and placed in two locations as shown in Figure (3).

Two different lengths of connecting hoses have been used $(60 \mathrm{~cm}$ and $110 \mathrm{~cm})$, both are provided with quick connection ends for easy connection and disconnection from different hydraulic elements.

An electric circuit has been used in system in order to control the hydraulic cylinder fluctuation action process. This circuit is divided into four control elements (control circuit, limit switches, delay timer, electric solenoid).

Two Fluid Technik pressure transducers model $(\mathrm{GmbH})$ of type PTA with pressure range $(0-100)$ bar with sensitivity $1 \%$ with measuring output range $(4-20 \mathrm{~mA})$, is chosen and installed on the experimental setup to measure the pressure of the hydraulic fluid at different stages, these sensors connect to the DAQ board using 2-conductor shielded cable.

The data acquisition system NI USB-6009 with 14 bits differential, 13 bits single-ended resolution, and $48 \mathrm{kS} / \mathrm{s}$ Sample Rate has been used to collect data to analyze the state of the system, this data is processed and filtered via the designed Labview software program version (8.6), then the data analyzed by using Matlab program to perform Fast Fourier Transformation (FFT) and displayed by A (2 GHZ) processor (core 2 Duo CPU) with (512 MB) installed memory (RAM) and (64-bit) operating system.

As well as the flow rates during process are measured by means of flow meters which display its values by two read out instruments.

\section{Measuring the Cylinder Pressure and Frequency Fluctuation}

The measurements will be carried out for different cases as

1- Measurements of pressure pulsation at both sides A and B, Figure (1).

2- Two accumulators are positioned at both sides of the cylinder (Case 1) Figure (3)

3- An accumulator is positioned at the hydraulic pump outlet (Case 2) Figure (3)

4- Changing the pre-charging pressure value of accumulator.

The circuit configuration for pressure pulsation at both sides of the hydraulic cylinder is shown in Figure (1) as well as the experimental result is shown in Figure (2), where

PT1... is the pressure transducer No. 1

PT2 ....is the pressure transducer No. 2

Because of similarity of double rod double action hydraulic cylinder used in this study it is clear from results shown in Figure (2) (a and b) that it is sufficient to use one pressure transducer at one side of the hydraulic cylinder and using the other one at the nearest point from the pump, so the pressure transducers will be put as shown in Figure (3) as case (1) and case (2) respectively. 


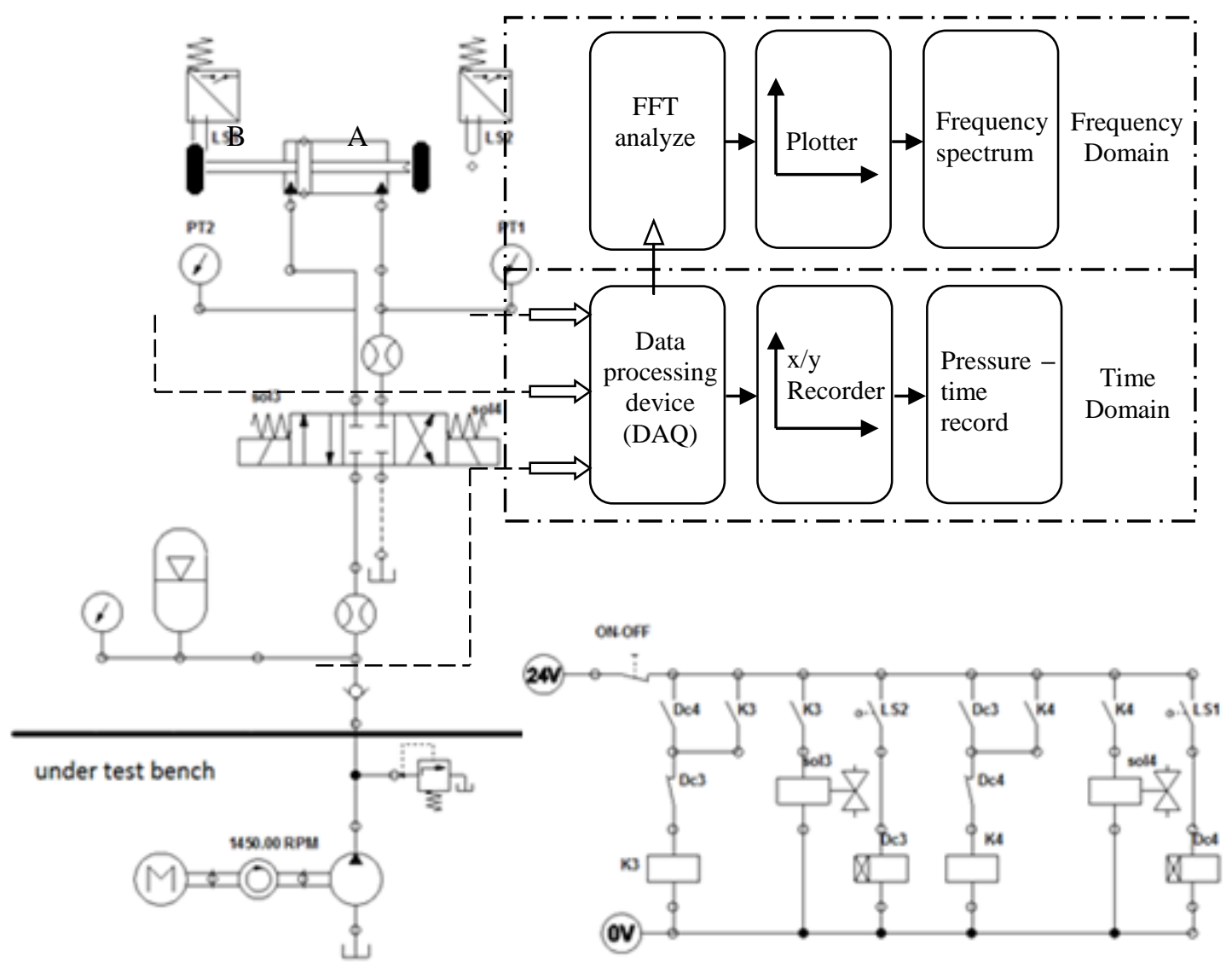

Electric control circuit

Figure (1) Test rig circuit with two pressure transducers at both sides of cylinder
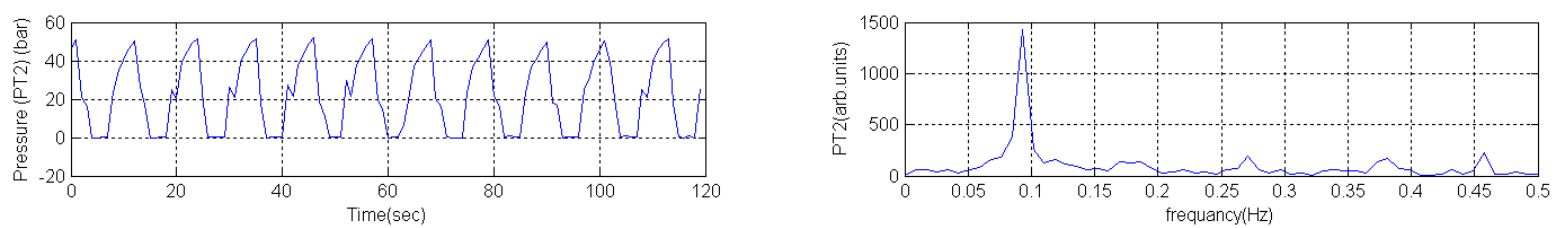

(a) Pressure variation in time and frequency domains from (PT2)
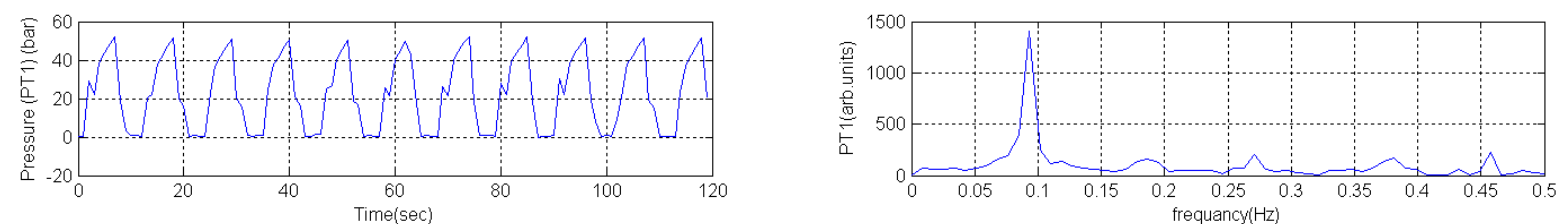

(b) Pressure variation in time and frequency domains from (PT1)

Figure (2) Pressure pulsation at both sides of the hydraulic cylinder 


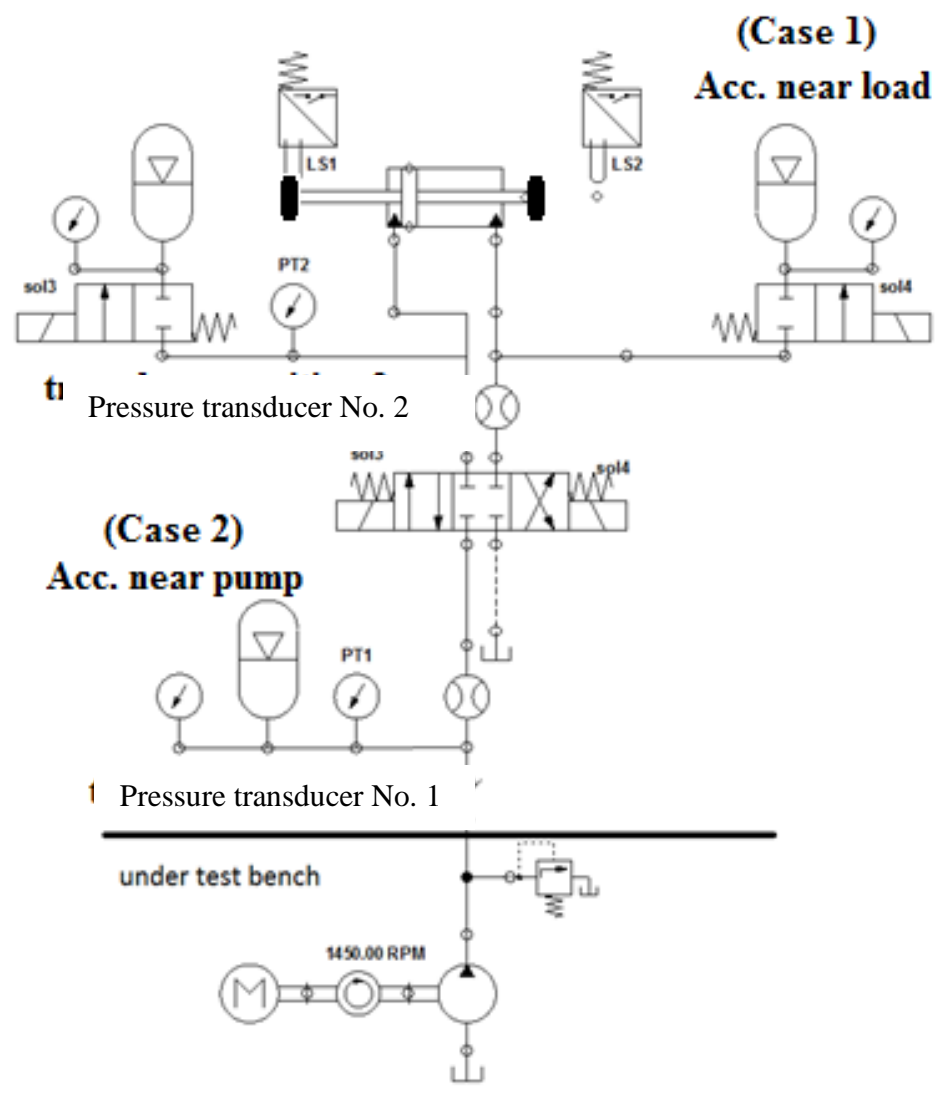

Figure (3) Test rig circuit for pressure transducers and accumulator positions

If the accumulator location is needed to be downstream the directional control valve so it is needed to be installed on both sides of the valves as case (1).

Figures (4 and 5) illustrate the signal results in time domain and frequency domain for three cases (case 1, case 2, case without accumulator); Figure (4) for pressure transducer No.2, Figure (5) for pressure transducer No.1.

It is shown from Figure (4) (a) and (b) that the pressure value in case 1 is (32.4 bar) and the pressure value in case 2 is ( 40 bar), so it is clear that when the accumulator is located near of the load, the pressure value has been reduced by $19 \%$.

\section{- Effect of Changing Pre-Charging Pressure of Accumulator}

For constant cylinder stroke and constant delay time ( $2 \mathrm{sec}$ ), many cases have been done by changing the pre-charging pressure value of accumulator from 10 to 20 bar at different accumulator location. For simplicity, cases study of 10 bar and 20 bar only illustrated in this paper.

\section{Results and Discussion}

The results of comparison between the systems with/without accumulator showed that the insertion of the accumulator has damped the pressure noise frequencies appeared in which the pressure and FFT domains showed.

This may enhance the system performance such that reduces the fluctuating in the pump pressure; prevent pump and connections from being damaged, keeping the system frequency 
away from the natural frequency of the system and reducing the insertion of flexible connections in frequent loads, all these positive issues may lead to reduce the noise and vibration of such field systems which in turn the system performance.

Also it is clear, as shown in Figures (4 and 5), that the accumulator results in absorption of some pressure waves that neglect some frequencies produced in the system, and reduces some of ripples of pressure fluctuations produced by the movement of the system and also reduces the transfer of these fluctuations to resonating devices, acting as hydraulic spring to dampen shocks and vibrations. Therefore the noise and vibration levels are reduced. In addition, by using accumulators, components which are sensitive to pressure oscillations ( e.g. hydraulic pumps) may be protected from being damaged and system service life is increased.

Also by using accumulator, the negative pressure peaks or pressure shocks produced if a column of moving fluid is suddenly stopped as we have in our study when fluctuating loads are applied may be avoided which may for example damage the pressure line filters or pump in the hydraulic system.
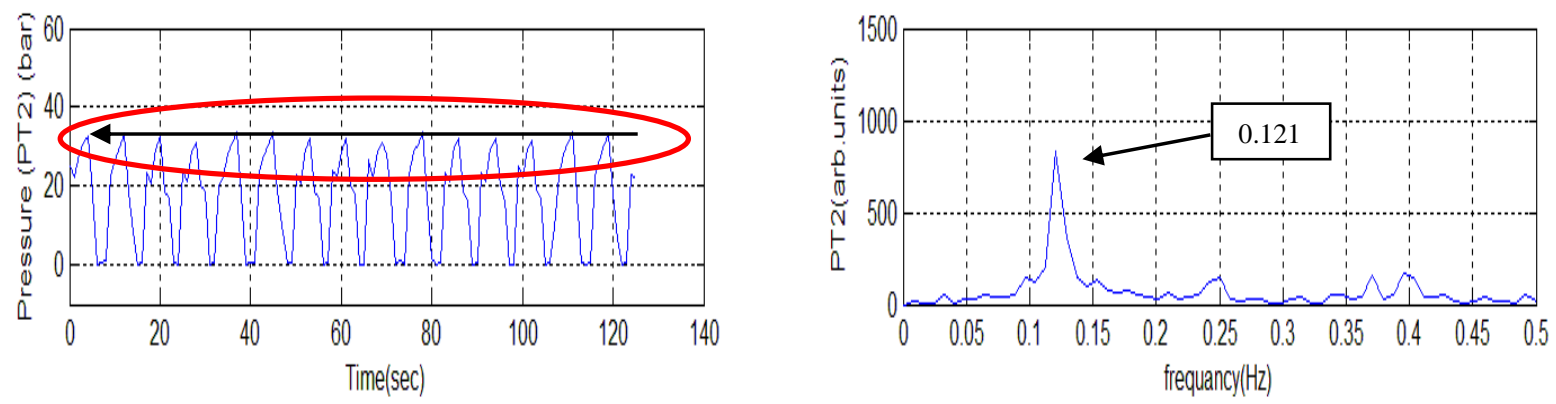

(a) Results of pressure transducer No.2 with two accumulators (Case 1)
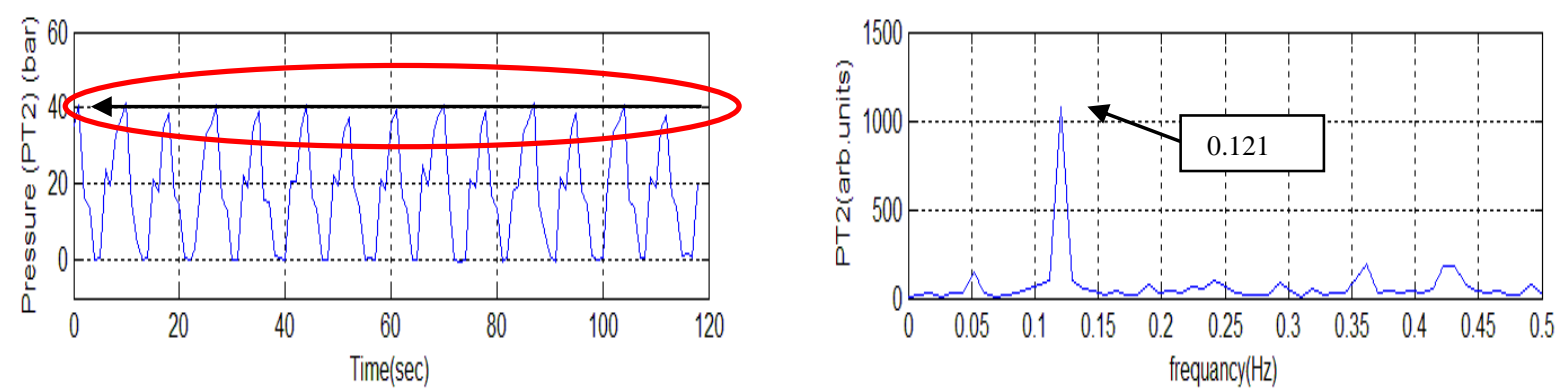

(b) Results of pressure transducer No.2 with an accumulator (Case 2)
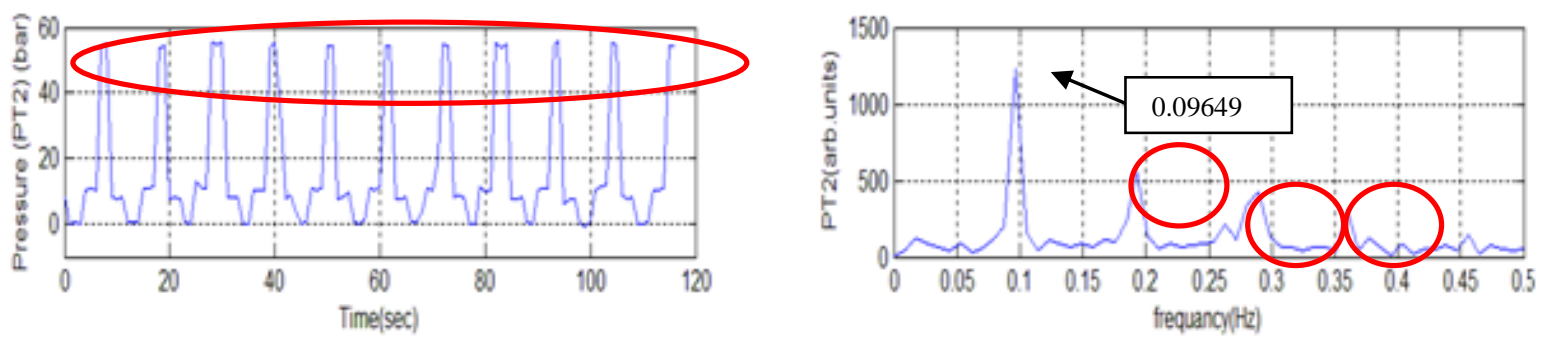

(c) Results of pressure transducer No.2 without accumulator

Figure (4) Results of pressure values and frequencies at pressure transducer No.2 

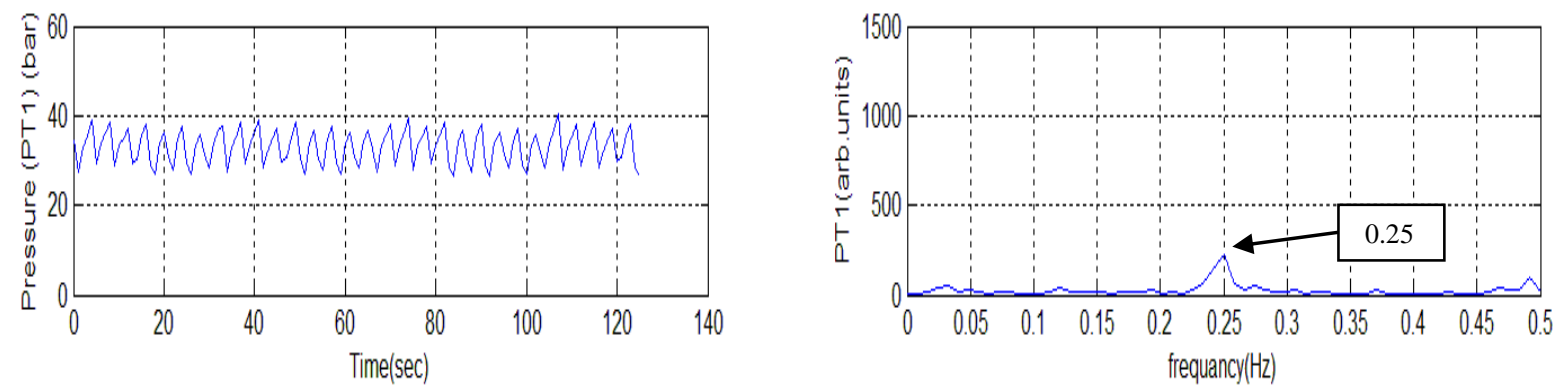

(a) Results of pressure transducer No.1 with two accumulator (Case 1)
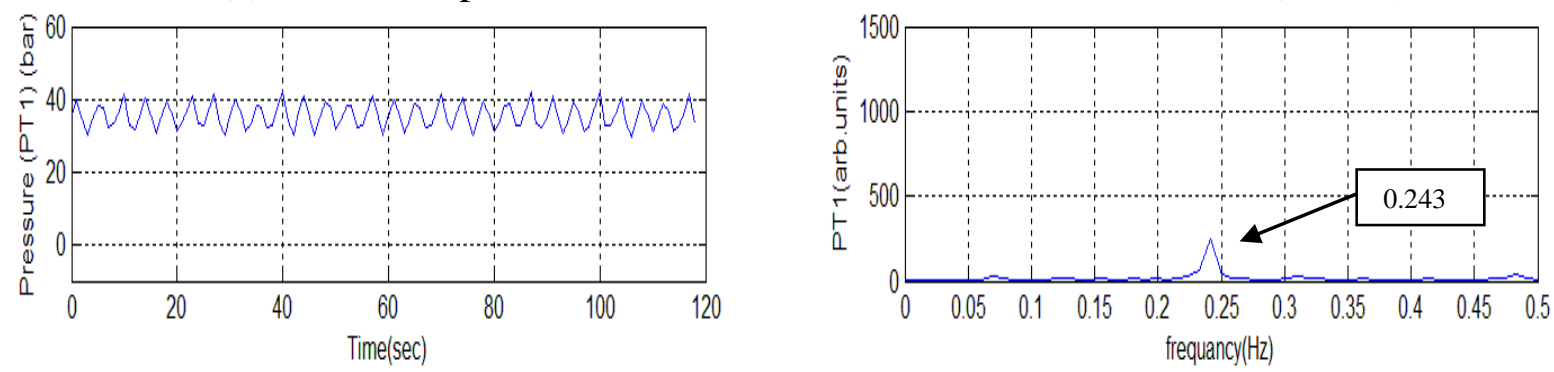

(b) Results of pressure transducer No.1 with an accumulator in Case (2)
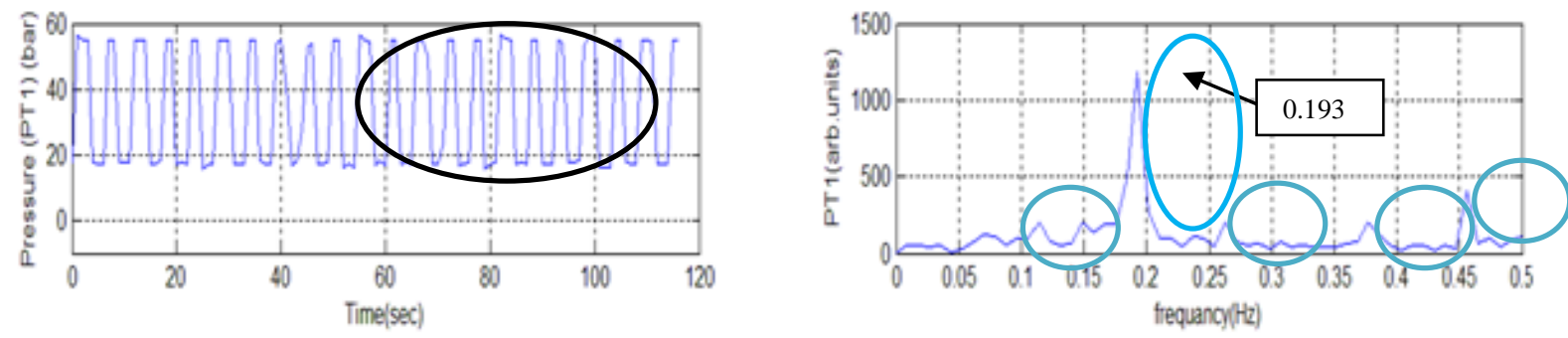

(c) Results of pressure transducer No.1 with an accumulator in Case (2)

Figure (5) Results of pressure values and frequencies at pressure transducer No.1

- For changing the pre-charge pressure of accumulator in case 1:
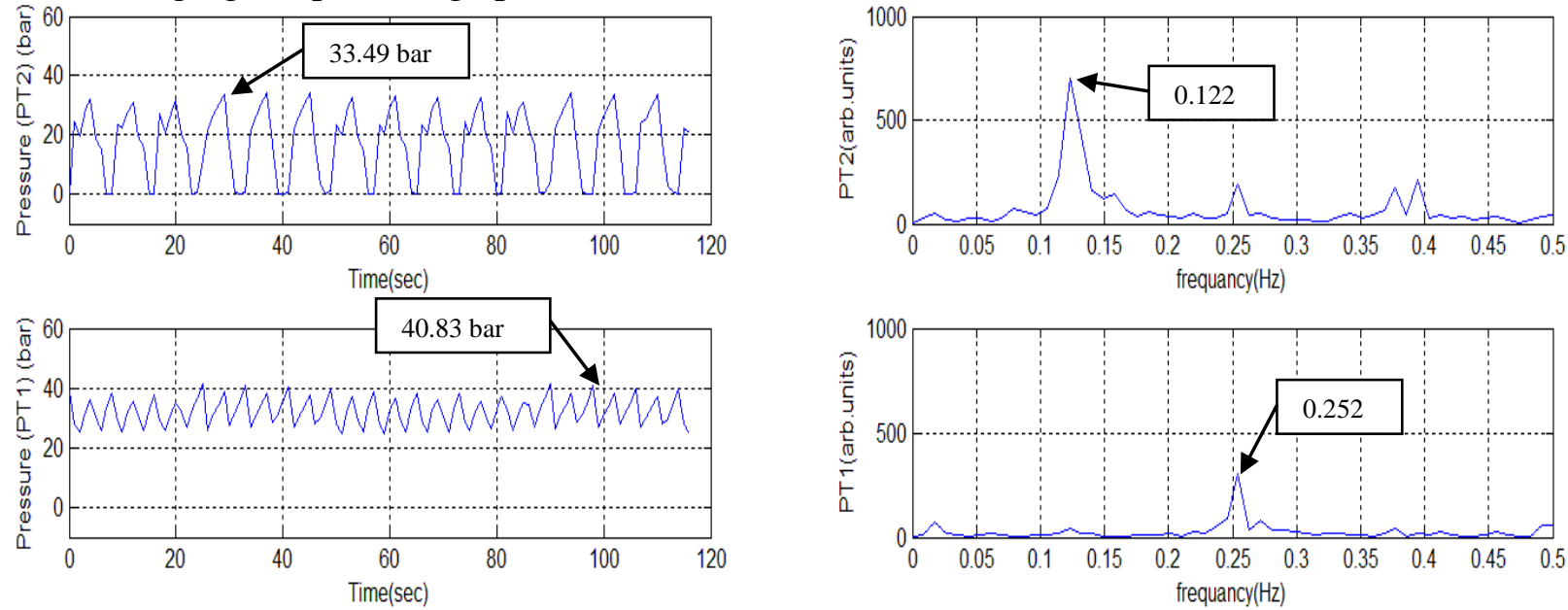

Figure (6) The accumulator pre-charge value is (10 bar) 

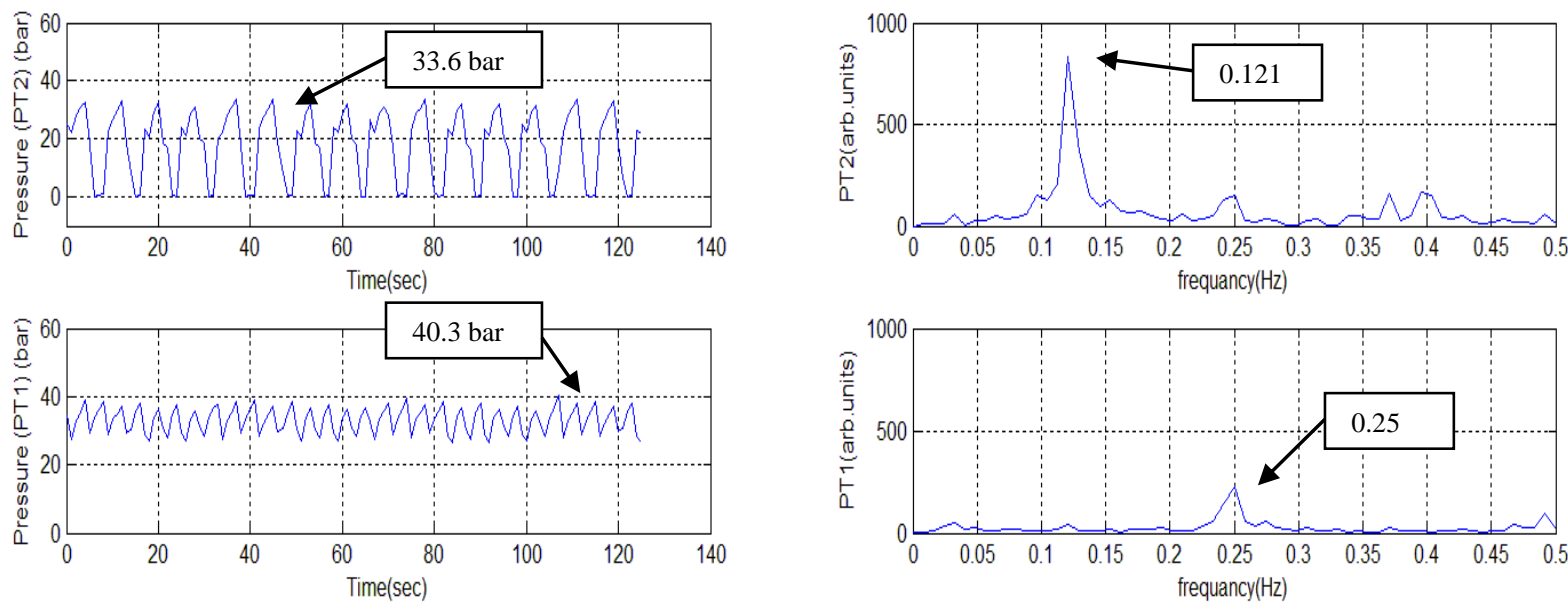

Figure (7) The accumulator pre-charge value is (20 bar)

- For changing the pre-charge pressure of accumulator in case 2:
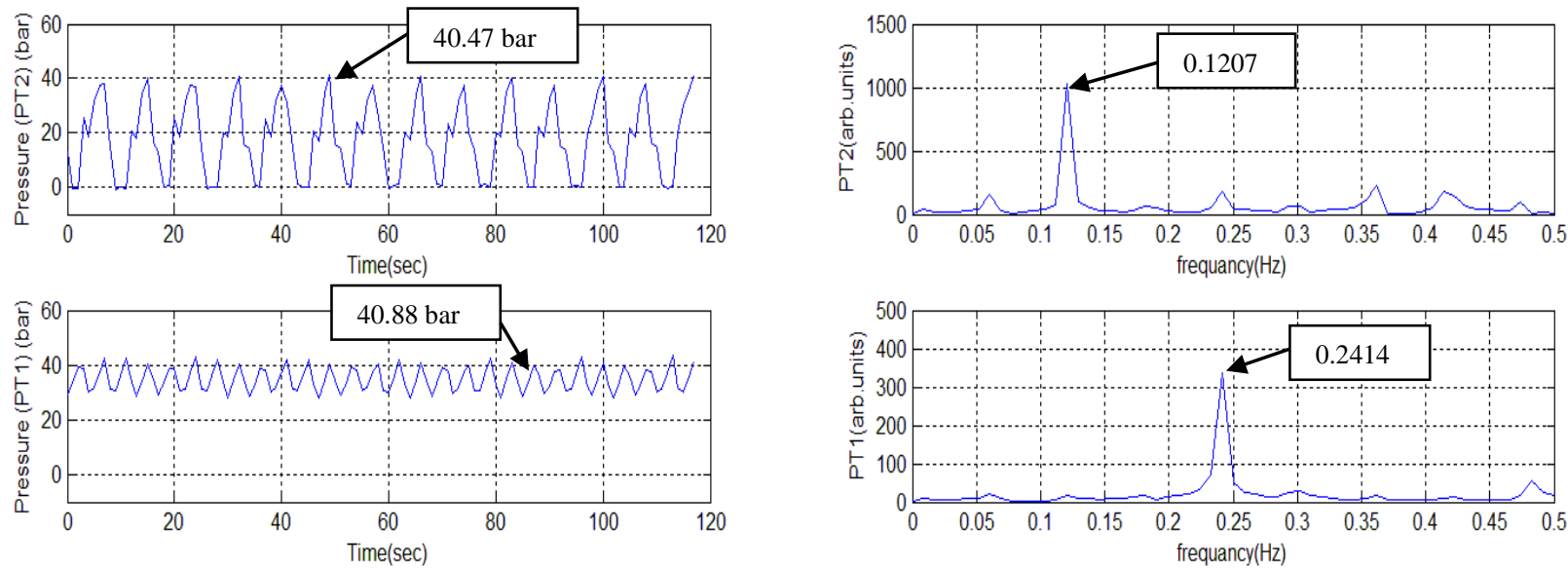

Figure (8) The accumulator pre-charge value is (10 bar)
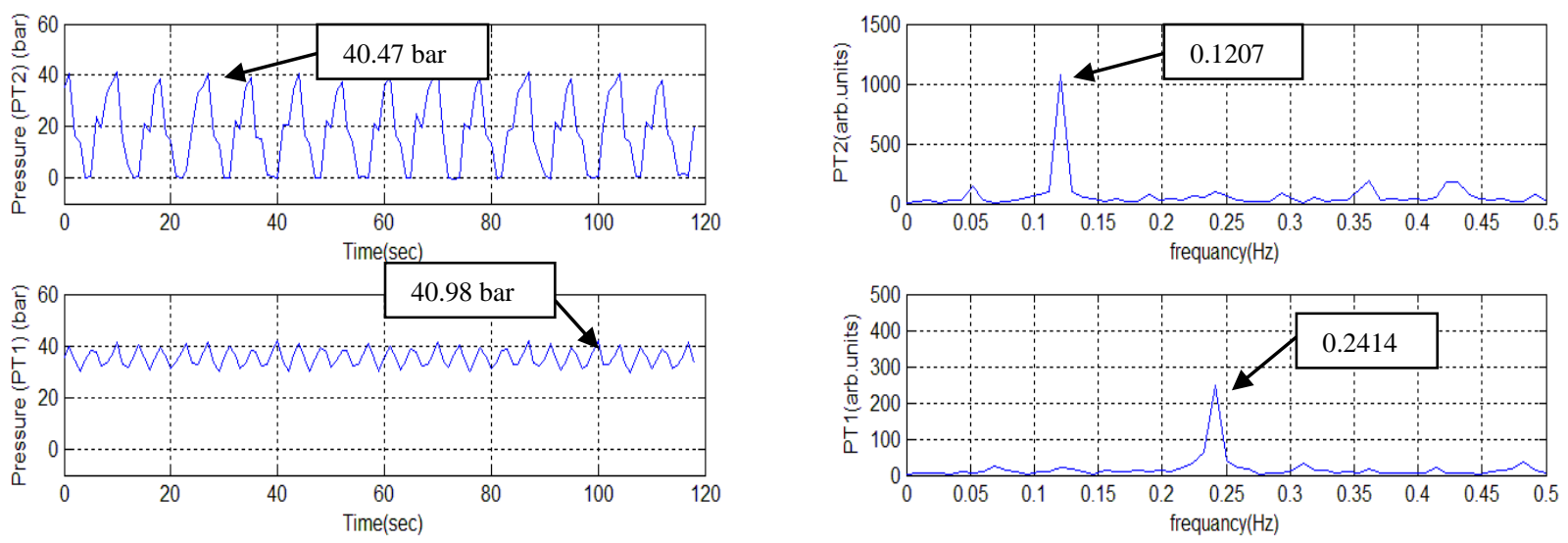

Figure (9) The accumulator pre-charge value is ( 20 bar) 
For changing the pre-charge pressure of accumulator if the accumulator location is near from load, case 1, it is observed from Figures (6 and 7) that there is no effective change in pressure values or in frequencies for this range of pressure.

Also For changing the pre-charge pressure of accumulator if the accumulator location is down near from pump, case 2, it is observed from Figures ( 8 and 9 ) that there is no effective change in pressure values or in frequencies for this range of pressure.

When the accumulator position is changed the results showed that the peak pressure values were reduced by $19 \%$ by using two accumulators at both sides of actuator, although the frequencies still the same, so the number of fluctuations will not change this mean that the periodicly motion of S-type swing pipe of a concrete pump equipment dosn't affected by the accumulator position.

\section{Conclusions}

Shifting of the natural frequencies of a system is often a very efficient step, which can be implemented by hydraulic dampers as accumulators.

Using of accumulator in hydraulic system will enhance the system performance in such that reduces the fluctuating in the pump pressure which leads to reduce the noise and vibration in all the system. The accumulator absorbed some pressure waves that neglect some frequencies produced by the movement of the system and also reduces the transfer of these fluctuations to resonating devices that may be damage the system.

Components which are sensitive to pressure oscillations (e.g. hydraulic pumps) may be protected from being damaged and system service life is increased by using accumulator.

When the accumulator position is at nearest point of the frequent load instead of the common position at the nearest point of the pump , the peak pressure values were reduced by $19 \%$ at this work condition but the frequencies still the same and the system timing will not affected.

\section{References}

[1] Bernd Seidl Gerhard Vetter, "Pressure pulsation damping methods for reciprocating pumps," tenth international pump users Texas A\&M University, 1993, pp. 25-39.

[2] Josef Mikota, "A novel, compact pulsation compensator to reduce pressure pulsation in hydraulic systems, " world scientific, vol. 69, 21 August 2001.

[3] Mika Ijas, "Damping of Low Frequency Pressure Oscillation," Doctor of Technology, Tampere University of technology, Finland, March 2007.

[4] Yangdong He, "Auxiliary energy-accumulation and flow enhancement Device for hydraulic system of concrete pump," United States Patent US 2010/0129250 A1, May 2010.

[5] Dennis J Reimer, "Hydraulics" vol. FM 5-499. Washington, DC: Department of the Army, March 1997.

[6] Liviu Vaida Claudia Kozma "Improvement in hydraulic impact mechanisms controlled by rotatable distributors," Hidrulica, vol. 1, pp. 19-26, 2013.

[7] Tamer Menshawy, "Investigation of dynamic behavior of an electro-hydraulic proportional system," Master of science in mechanical engineering, Mechanical power \& energy, Military Technical College, Cairo, 2006. 